\title{
Wallerian-Like Degeneration of Central Neurons After Synchronized and Geometrically Registered Mass Axotomy in a Three-Compartmental Microfluidic Chip
}

\author{
Devrim Kilinc • Jean-Michel Peyrin • \\ Vanessa Soubeyre • Sébastien Magnifico • \\ Laure Saias • Jean-Louis Viovy • Bernard Brugg
}

Received: 3 August 2009/Revised: 15 October 2009/Accepted: 12 January 2010/Published online: 17 February 2010

(C) The Author(s) 2010. This article is published with open access at Springerlink.com

\begin{abstract}
Degeneration of central axons may occur following injury or due to various diseases and it involves complex molecular mechanisms that need to be elucidated. Existing in vitro axotomy models are difficult to perform, and they provide limited information on the localization of events along the axon. We present here a novel experimental model system, based on microfluidic isolation, which consists of three distinct compartments, interconnected by parallel microchannels allowing axon outgrowth. Neurons cultured in one compartment successfully elongated their axons to cross a short central compartment and invade the outermost compartment. This design provides an interesting model system for studying axonal degeneration and death mechanisms, with a previously impossible spatial and temporal control on specific molecular pathways. We provide a proof-of-concept of the system by reporting its application to a well-characterized experimental paradigm, axotomy-induced Wallerian degeneration in primary central neurons. Using this model, we applied localized central axotomy by a brief, isolated flux of detergent. We report that mouse embryonic cortical
\end{abstract}

Electronic supplementary material The online version of this article (doi:10.1007/s12640-010-9152-8) contains supplementary material, which is available to authorized users.

D. Kilinc $\cdot$ J.-M. Peyrin $\cdot$ V. Soubeyre $\cdot$ S. Magnifico

B. Brugg $(\square)$

Laboratoire de Neurobiologie des Processus Adaptatifs, UMR 7102 CNRS/UPMC, Univ. P. et M. Curie, Bat B, 6eme Etage, Case courrier 12, 9 Quai St. Bernard,

75252 Paris, France

e-mail: bernard.brugg@snv.jussieu.fr

L. Saias · J.-L. Viovy

Laboratoire Physicochimie-Curie, UMR 168 Institut Curie/

CNRS/UPMC, Paris, France neurons exhibit rapid Wallerian-like distal degeneration but no somatic death following central axotomy. Distal axons show progressive degeneration leading to axonal beading and cytoskeletal fragmentation within a few hours after axotomy. Degeneration is asynchronous, reminiscent of in vivo Wallerian degeneration. Axonal cytoskeletal fragmentation is significantly delayed with nicotinamide adenine dinucleotide pretreatment, but it does not change when distal calpain or caspase activity is inhibited. These findings, consistent with previous experiments in vivo, confirm the power and biological relevance of this microfluidic architecture.

Keywords Central nervous system .

Wallerian degeneration - Microfluidic cell culture

In vitro axotomy $\cdot$ Axonal degeneration

\section{Introduction}

Axon elimination or pruning without neuronal death is a process to selectively remove redundant neuronal connections during normal development of the nervous system. Pathological degeneration, on the other hand, may occur in various neurological diseases (Luo and O'Leary 2005) or in response to injury (Houle and Tessler 2003). Axotomy, the severing of the axon from the cell body, initiates a series of pathological events, termed Wallerian degeneration, which result in the degeneration of the distal stump (Griffin et al. 1995; Waller 1850). In embryonic neurons axotomy induces apoptosis; however, in the adult neuron apoptosis is only induced if the axotomy site is close to the cell body (Aguayo et al. 1991). Wallerian degeneration is characterized by a rapid breakdown of the axonal cytoskeleton distal to the severing site, following a latency of variable 
duration, leading to axonal fragmentation and subsequent phagocytosis of axonal debris by recruited glial cells and macrophages (Beirowski et al. 2005). Axonal degeneration may also occur in a distal-to-proximal manner due to various neurological diseases such as amyotrophic lateral sclerosis, spinal muscular atrophy or peripheral neuropathy. In its final stages, this so-called "dying back" degeneration exhibits axonal fragmentation similar to Wallerian degeneration (Griffin et al. 1995; Raff et al. 2002). In addition, neurodegenerative diseases such as Alzheimer's, Parkinson's, and Huntington's cause axonal degeneration which is important in the occurrence of clinical symptoms (Bossy-Wetzel et al. 2004). Wallerian-like degeneration following stroke (Kuhn et al. 1989) and traumatic axonal injury (Buki and Povlishock 2006) are well documented; however, it is not clear if axotomy-induced Wallerian degeneration is relevant for studying central nervous system (CNS) disease mechanisms. In order to understand the complex mechanisms involved in the injury-induced axonal degeneration, and to test potential therapeutic approaches, we have developed an experimental model system that enables simultaneous in vitro axotomy of a large number of primary CNS neurons, and provides exclusive access to proximal and distal segments before and after axotomy.

Axotomy in the peripheral nervous system (PNS) has been studied extensively in vivo by transection or crush of the rodent sciatic nerve (Hanz et al. 2003; Beirowski et al. 2009; Glass et al. 1993). In the CNS, in vivo axotomy has been conducted by crushing sensory neurons in sea slug (Ambron et al. 1995), by transecting individual axons in mouse spinal cord using fine needles (Kerschensteiner et al. 2005), by applying fluid percussion trauma to rat brain (Singleton et al. 2002), or by transecting the optic nerve (Aguayo et al. 1991). Recently, single axons of the nematode have been cut by vaporizing $5-\mu \mathrm{m}$ segments with light from a femtosecond pulsed laser (Yanik et al. 2004). CNS axotomy has also been studied in vitro by transecting axons originating from chick dorsal root ganglion (DRG) explants with fine glass capillaries (Gallo 2004), mouse DRG explants with needles (Araki et al. 2004), and rat cortical neurons with glass microelectrodes (Mandolesi et al. 2004). Very recently, cortical and DRG axons were cut within microfluidic channels using light from a femtosecond pulsed laser with high precision (Kim et al.2009). In vitro axotomy models may be preferred over in vivo models due to their practical advantages and the fine control on the target axon population they provide. However, available in vitro models require specialized tools and skills as well as considerable experiment time, since each axon has to be selected and microdissected individually. In addition, their interpretation is limited by the intrinsic randomness and heterogeneity of axons grown in vitro. An improved experimental model system that simplifies in vitro axotomy and provides better control on the geometry is thus highly needed.

Microfluidics, the manipulation of small liquid volumes $\left(10^{-9}-10^{-18} 1\right)$ using channels with dimensions of tens to hundreds of micrometers, have been recently introduced to neurobiology research (Weibel et al. 2005). Since neurons are particularly sensitive to environmental cues such as substrate properties (Fan et al. 2002) and temperature (Pearce et al. 2005), microfluidic culture systems for neurons have additional design constraints as compared to systems for the culture of other cell types (El-Ali et al. 2006). Microfluidic systems have already been employed for studying neuronal stem cell differentiation (Chung et al. 2005; Nakashima and Yasuda 2007), pharmacological interactions (Ravula et al. 2007), electrophysiology (Morales et al. 2008; Morin et al. 2006), and neuron-based biosensing (Sato et al. 2006). Applying mechanical constraints to axon outgrowth (Francisco et al. 2007) and testing the effect of biochemical factors (Vahidi et al. 2008) have been other recent uses of microfluidic technology in neuroscience research. Axonal regeneration following mechanical injury has been demonstrated in a microfluidic chip based on subcellular compartmentalization achieved by fluidic isolation (Taylor et al. 2005). Although this particular injury model provides a useful tool to study axonal regeneration in CNS neurons, it does not allow the study of distal axon degeneration, since the distal segments of axons are completely destroyed at the time of mechanical injury (Taylor et al. 2005). New experimental model systems based on subcellular compartmentalization are thus necessary to elucidate disease- or injury-induced axonal degeneration mechanisms.

In this article, we report a novel, three-compartmental neuronal culture system for inducing local axotomy and treating distal and proximal axons independently. In contrast to available in vitro CNS axotomy models, this novel design enables simultaneous axotomy of a large number of axons at a similar distance from the cell body, thereby providing a time and cost effective model for CNS axotomy studies. Moreover, exclusive access to proximal and distal compartments before and after axotomy, provided by microfluidic isolation, enables the examination of various molecular mechanisms. Spatial and temporal dissociation of the molecular mechanisms involved in the axotomyinduced degeneration cascade provides a deeper understanding of the axon degeneration and its role in neurodegenerative diseases. Using this novel model system we applied central axotomy to cultured primary CNS neurons and investigated major molecular pathways associated with neurodegeneration. Our findings indicate that central axotomy induces Wallerian-like distal axon degeneration in CNS neurons as previously reported in PNS neurons. We 
thus confirm that our in vitro axotomy model can be a relevant model system for studying CNS axon degeneration.

\section{Materials and Methods}

\section{Circuit Design and Fabrication}

We developed a novel tri-compartmental microfluidic neuron culture system (Fig. 1) based on a previously established bi-compartmental design, using microchannels for directed axon outgrowth and compartmentalization of the culture (Taylor et al. 2005). The three-compartmental neuronal circuits were fabricated using standard photolithography methods (Weibel et al. 2007). The circuit design consists of somatic and axonal compartments, each $1 \mathrm{~mm}$ long, and a $100 \mu \mathrm{m}$ long central compartment. The compartments are connected to each other by $10 \mu \mathrm{m}$ wide,
$3 \mu \mathrm{m}$ high, and $450 \mu \mathrm{m}$ long parallel microchannels that are $20 \mu \mathrm{m}$ apart from each other (Fig. 1). The height of all compartments is $55 \mu \mathrm{m}$. In order to fabricate masters with elements of two different depths, two layers of photoresist were used. Silica wafers were heated to $150^{\circ} \mathrm{C}$ and activated by plasma treatment for $30 \mathrm{~s}$. The first layer of photoresist SU8-2002 (Microchem, Newton, USA) was spin-coated on the wafer at $2000 \mathrm{rpm}$ followed by a softbake at $65^{\circ} \mathrm{C}$ for $2 \mathrm{~min}$ and at $95^{\circ} \mathrm{C}$ for $4 \mathrm{~min}$. The master is then exposed to UV light through an optic mask containing the microchannel features. Following the hardbake, microchannels of 2-3 $\mu \mathrm{m}$ height were developed in SU8 developer (Microchem). A layer of dry film SY355 (Elga Europe, Milano, Italy) was then laminated on the wafer at a thickness of $55 \mu \mathrm{m}$ at $70^{\circ} \mathrm{C}$. A second mask containing compartment geometries was aligned over the SU8 patterns and exposed to UV light. The second layer was then baked at $120^{\circ} \mathrm{C}$ for $5 \mathrm{~min}$ and developed in BMR
Fig. 1 (a) Technical drawing of the three-compartmental microfluidic circuit (dimensions given in $\mu \mathrm{m}$ ). (b) A photograph showing fluidic isolation of somatic (red), central (black), and axonal (green) compartments. (c) Threedimensional representation of the circuit with 1-mm high reservoirs. (d) Detail image showing the boxed area in $\mathrm{C}$
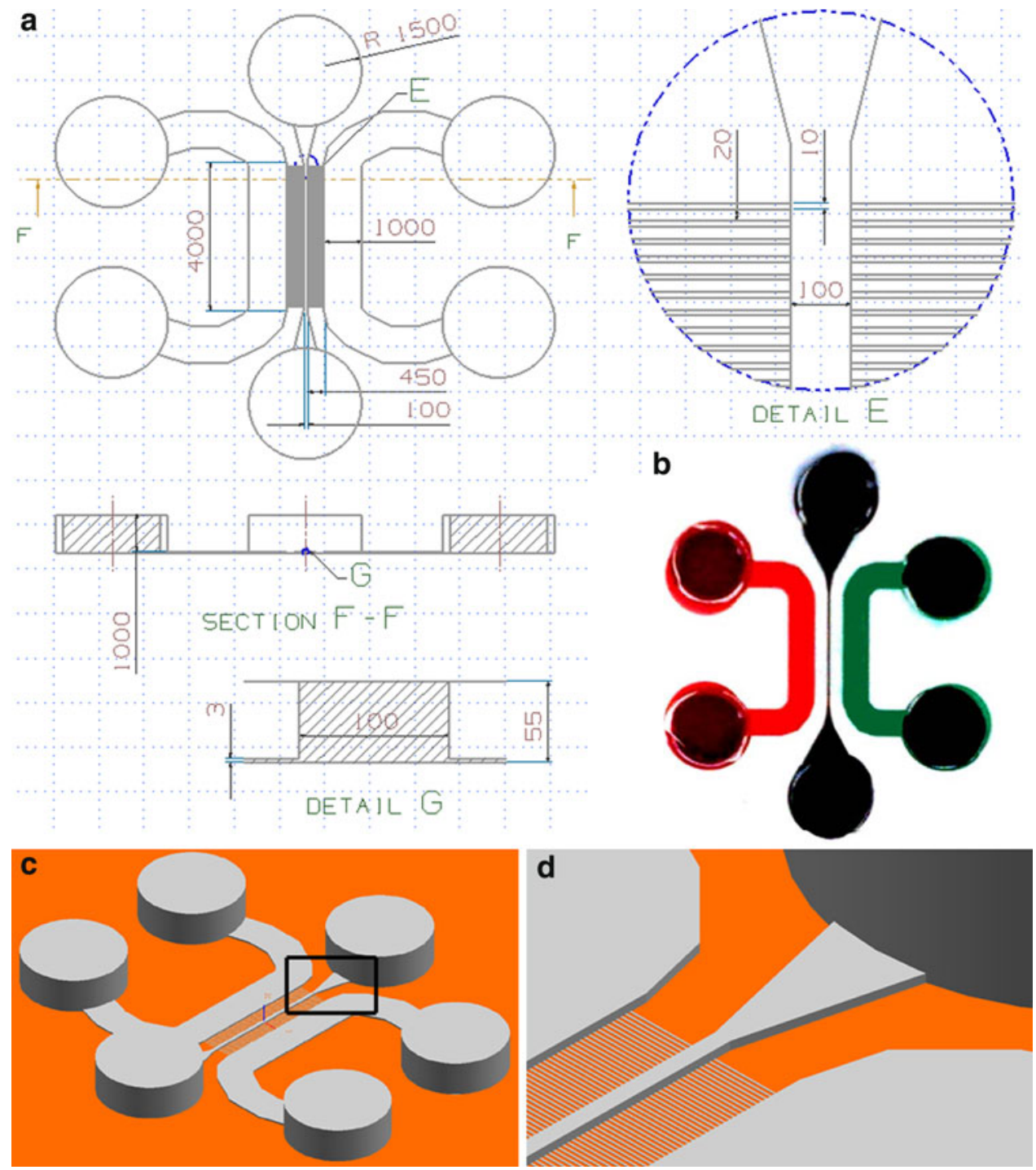
developer (Elga Europe) and then rinsed with isopropanol. 3-4 mm thick Polydimethylsiloxane (PDMS; Dow Corning, Midland, USA) pads were cast at $70^{\circ} \mathrm{C}$ for $2-3 \mathrm{~h}$ using masters which contain four circuits with identical design pattern. Through holes were punched out with a $4 \mathrm{~mm}$ diameter metallic biopsy punch to create reservoirs for culture medium. The punched area was slightly larger than the well area of the circuit design and enclosed it completely. The pads were then irreversibly bonded to glass coverslips (Menzel Glass, Braunschwein, Germany) by activating their surfaces with an air plasma generator (Diener Electronic, Nagold, Germany) and manually placing them on top of coverslips whose surfaces were also activated. The circuits were sterilized with UV light and treated with $10 \mu \mathrm{g} / \mathrm{ml}$ Poly-D-lysine (Sigma-Aldrich, Saint Louis, USA) overnight.

\section{Neuron Culture and Treatments}

Mouse cortices at embryonic day 14 were dissected and dissociated as described elsewhere (Willaime et al. 2001). Neurons were seeded at a density of $10^{5}$ cells $/ \mathrm{cm}^{2}$ which yields to approximately 4000 neurons in the somatic compartment. DMEM containing $4.5 \mathrm{~g} / \mathrm{l}$ D-glucose and pyruvate (Invitrogen, Carlsbad, USA) supplemented with 10\% FBS (PAA Laboratories, Pasching, Germany) and 2\% B27 (Invitrogen) was used as culture medium. The medium was replaced every 3 days prior to experiment and the cultures were kept in an incubator at $37^{\circ} \mathrm{C}$ and $5 \% \mathrm{CO}_{2}$. The experiments were conducted at 7-8 days in vitro. Calpain inhibitor N-Acetyl-Leu-Leu-Nle-CHO (ALLN; $10 \mu \mathrm{M}$; Calbiochem), general inhibitor of caspase Z-V-AD-Fluoromethyl ketone (z-VAD; $20 \mu \mathrm{M}$; R\&D Systems, Minneapolis, USA), and $\beta$-Nicotinamide adenine dinucleotide hydrate ( $\beta$ NAD; $5 \mathrm{mM}$; Sigma) were used as treatment paradigms.

\section{Axotomy Procedure}

Axotomy of axons was conducted by flowing $15 \mu \mathrm{lmed-}$ ium with $0.1 \%$ saponin through the central compartment for $2 \mathrm{~min}$ while over-pressurizing axonal and somatic compartments (with $40 \mu \mathrm{l}$ medium in each) to prevent flow of the detergent into the axonal microchannels. At the end of 2 min period, saponin containing medium was removed and the central compartment was rinsed with culture medium for another $5 \mathrm{~min}$, followed by the addition of new culture medium.

\section{Flow and Diffusion Experiments}

A series of flow and diffusion experiments were conducted in order to verify that (i) axotomy is localized to the central compartment and (ii) long-term treatments remain confined in the individual compartments they are addressed to. Simulation of the axotomy procedure was conducted by flowing fluorescent microspheres with $1 \mu \mathrm{m}$ diameter (Invitrogen) suspended in saponin-PBS solution and tracking the flow using video microscopy. In separate experiments, Alexa Fluor 555-conjugated goat anti-rabbit Immunoglobulin $\mathrm{G}$ (IgG; 1:100; Invitrogen) was used as a tracer to measure the extent of particle diffusion both during axotomy and during long-term treatments. The concentration-fluorescence relation was established by imaging compartments filled with different dilutions of the tracer, and a linear relationship between fluorescence signal and tracer concentration was found in the two-log range $\left(R^{2}>0.99\right)$. Three circuits per experiment and three regions per compartment were imaged at designated time points to quantify the diffusion of the marker (i) from central compartment to the somatic compartment during axotomy and (ii) from the axonal compartment to the central and somatic compartments during long-term treatment. A nonspecific signal was acquired by using the DAPI filter and subtracted from the Alexa Fluor 555 signal to eliminate the variation in the signal intensity due to local illumination differences. Axotomy was simulated by adding $100 \%$ tracer to the detergent solution and using the same volume combination as in the axotomy experiments. Images were acquired at 2, 5, and $10 \mathrm{~min}$ following the addition of marker-containing detergent solution into the reservoir. Long-term diffusion of molecules was simulated by adding the tracer into the axonal compartment and balancing the circuit by placing equal volumes to other reservoirs. Images were acquired at $1,2,4$, and $8 \mathrm{~h}$ following the addition of the marker to the axonal compartment. In addition, a multi-tracer time-lapse simulation of axotomy was conducted by placing $500 \mathrm{nM}$ CellTracker Blue-CMAC (Invitrogen; MW $=209.63 \mathrm{Da}$ ) to the side compartments and $0.5 \mathrm{mg} / \mathrm{ml}$ Texas Red Dextran (Invitrogen; $\mathrm{MW}=10 \mathrm{kDa}$ ) in the detergent solution.

\section{Measurement of Calpain Activity}

To detect calpain activity in living neurons, the fluorogenic calpain substrate 7-amino-4-chloromethylcoumarin, $t$-BOC1-leucyl-1-methionine amide (t-BOC; Invitrogen) was used. Steady state calpain activity results in an increase in the t-BOC fluorescence due to the accumulation of fluorescent end product of the calpain substrate. Changes in calpain activity can be detected by considering the rate of change in the signal intensity (Kilinc et al. 2009). Culture medium containing $10 \mu \mathrm{M}$ t-BOC was introduced to neurons following axotomy. Registered areas on microfluidic circuits were imaged twice with an interval of $10 \mathrm{~min}$ at $15-20 \mathrm{~min}$ and 25-30 min after the addition of t-BOC. The change in 
the $\mathrm{t}$-BOC signal intensity during the $10 \mathrm{~min}$ interval is measured for individual axons, and this value was used for comparing different experimental groups.

\section{Immunocytochemistry}

Neuron cultures were fixed with $4 \%$ Paraformaldehyde (Sigma) at designated time points for immunocytochemistry. Circuits were immunostained with anti-MAP2 (rabbit IgG, 1:300; Chemicom, Temecula, USA), monoclonal anti$\beta$-III-tubulin (mouse IgG, 1:300; Sigma) for cytoskeletal protein content and stained with Hoechst 33342 (1:25,000; Sigma) to reveal nuclei. In a separate experiment, circuits were also stained with anti-glial fibrillary acidic protein (GFAP, rabbit IgG, 1:500; DakoCytomation, Glostrup, Denmark), a marker for astrocytes. A subset of cultures was subjected to simultaneous fixing and extraction, a procedure that removes free tubulin from cytoplasm while fixing microtubules (Gallo and Letourneau 1999). These cultures were immunostained with FITC-conjugated monoclonal anti- $\alpha$-tubulin (Sigma) and stained with Alexa Fluor 555 Phalloidin (Invitrogen) to reveal microtubules and actin cytoskeleton, respectively. Images were acquired with an inverted epi-fluorescent microscope (Zeiss, Goettingen, Germany) equipped with a CoolSnap HQ2 camera (Roper Scientific, Ottobrunn, Germany) and using micro-manager image acquisition software (http://www.micro-manager.org).

\section{Quantification}

We analyzed axonal degeneration by comparing axonal tubulin staining among experimental groups. Cortical neurons require high seeding density; subsequently, a very high number of axons cross to the distal compartment making it difficult to quantify individual axons. Crossing of axons from central to distal compartment was quantified by measuring tubulin staining intensity of fixed cultures in defined regions at channel exits and calculating their ratio. We also developed an automated quantification method for axon fragmentation and cross-checked the automated results with manual counting results. Using a macro developed in NIH ImageJ software (http://rsbweb.nih.gov/ij/) that utilizes Otsu thresholding algorithm (Otsu 1979), and a particle analyzer algorithm of ImageJ, axonal regions with a circularity greater than 0.9 were determined. The total area of such regions was calculated, and this value was normalized by the total axonal area, which was measured from the thresholded image. This ratio, termed fragmentation index, is an indicator of the average axonal fragmentation level of the axons and is used in statistical comparisons. Image analysis steps are demonstrated for axonal images with indices of 0.005 , 0.083 , and 0.157 (corresponding to $<5 \%, 50 \%$, and $>95 \%$ fragmentation, respectively) in Supplementary Figure 1.
Somatic death was quantified by dividing the number of cell bodies with condensed bright staining of Hoechst dye by the total number of cell bodies. At least five images were analyzed per circuit for both axonal degeneration and somatic death quantification. Hoechst count was conducted blindly. The growth cone density was quantified by counting dense actin structures resembling normal growth cones and normalizing this number with the total axon area of the corresponding imaging field using tubulin co-staining.

\section{Statistical Analysis}

In statistical calculations, $N$ represents the number of individual circuits. Five randomly selected imaging fields were analyzed for each compartment. Data were expressed as mean \pm standard error of the mean (SEM). Statistical analysis was performed using one way analysis of variance (ANOVA), followed by Kramer's modification of Tukey's post hoc test to determine significance values between different experimental groups.

\section{Results}

\section{Experimental Control of Fluid Flow During Central Axotomy and Long-Term Treatment}

Liquids in microfluidic systems flow from high pressure to low pressure regions, as in all fluidic circuits. To achieve a controlled flow of a liquid through a compartment, a hydrostatic pressure gradient is created by placing different amounts of liquid in opposite reservoirs. In order to obtain a map of flow during our experiments, we first performed model experiments using fluorescent microspheres as tracers in the flowing liquid. Flow of the microspherecontaining detergent solution through the central compartment was obtained by preparing a volume difference of 15 vs. $0 \mu \mathrm{l}$ between the inlet and outlet of the central compartment, while side compartments were over-pressurized with $40 \mu \mathrm{l}$ PBS in both reservoirs (all reservoirs have the same cross-section, so hydrostatic pressures at the interface between a reservoir and the corresponding microchannel are proportional to the loaded volume). Images acquired during the first 180s of the flow demonstrate that the detergent solution does not enter into the microchannels. The 120s time point image was taken from the downstream portion of the central compartment; whereas at other time points, images were taken from the upstream portion (Fig. 2a; Supplementary Movie 1). In the downstream portion, the flow of the microspheres is limited to the central core (facing arrows) of the compartment, indicating a flow from the over-pressurized somatic and axonal compartments. A reverse experiment was conducted 
a
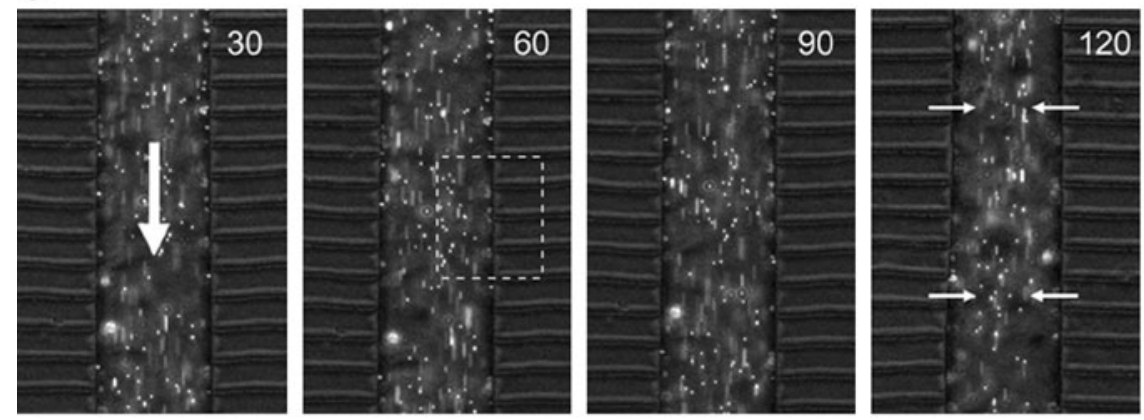

b

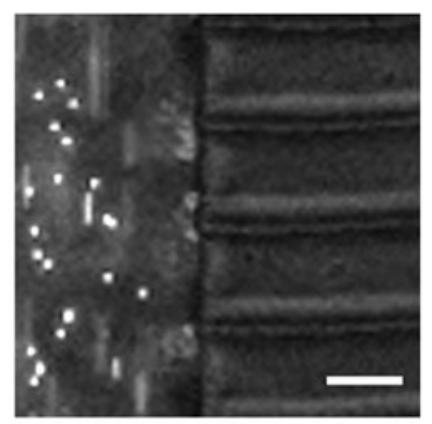

C

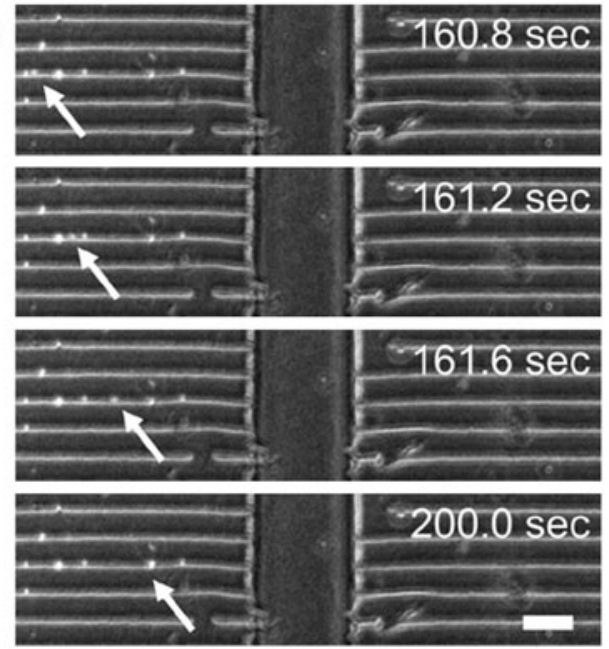

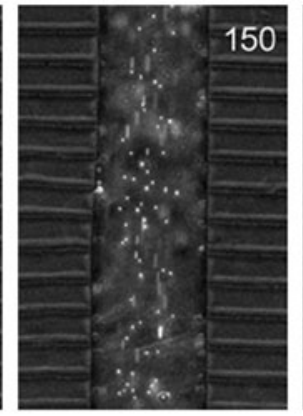

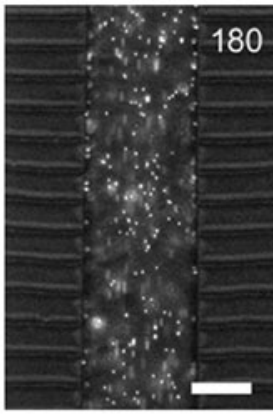

d
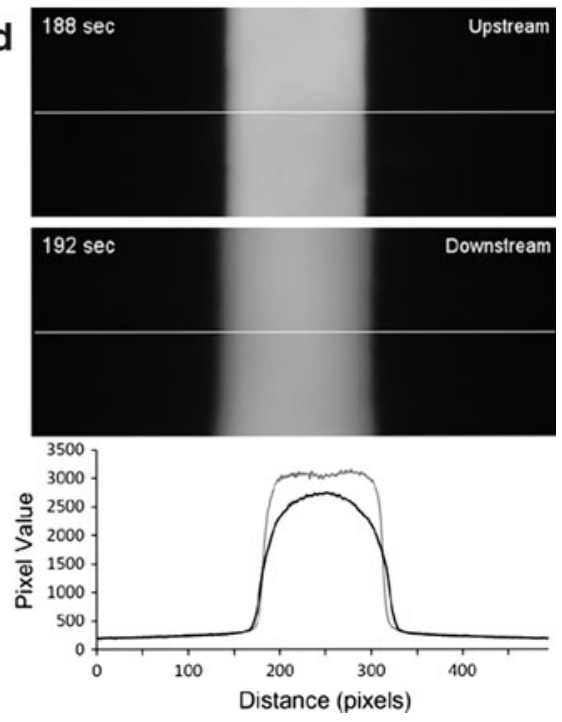

Fig. 2 Simulation of central axotomy procedure. (a) Pressure driven flow of the saponin solution through the central compartment is restricted to this compartment as visualized by fluorescent microspheres. In the downstream portion of the compartment (120 s time point), the flow is further restricted to the central core (facing arrows) due to the flow from opposing compartments. Scale bar $=50 \mu \mathrm{m}$. Numbers denote time after the initiation of flow in seconds. (b) Higher magnification image of the boxed region in A. Scale bar $=20 \mu \mathrm{m}$. (c) The reverse experiment with fluorescent microspheres placed in opposing compartments. Numerous microspheres can be seen in microchannels indicating flow towards the central

by placing the microspheres in side compartments and flowing microsphere-free detergent solution through the central compartment, using same the volume configuration described above. The movement of the tracers demonstrates a flow from the over-pressurized side compartments towards the central compartment. At 120s time point numerous tracer microspheres can be found in the microchannels, and occasional flow of microspheres can be observed at later time points (Fig. 2c).

Fluorescently labeled IgG and Dextran molecules were used as diffusion tracers. During axotomy simulation, there was no tracer diffusion away from the central compartment; however, the tracer concentration in the central compartment decreased linearly $\left(R^{2}>0.99\right.$; Table 1$)$. At the 3 min time point, tracer in the central compartment was observed compartment. Inter-compartmental flow persists even after 2 min, shown by the movement of additional microspheres (arrows). Vertical arrows denote flow direction. Scale bar $=50 \mu \mathrm{m}$. (d) Distribution of fluorescent tracer in the upstream and downstream portion of the central compartment 3 min after simulated axotomy. A plot of pixel values along the horizontal lines shows a rectangular profile for the upstream portion (gray plot) and a bell-like curve for the downstream portion (black plot); indicating that the detergent flow is focused into the core of the channel at the downstream portion due to the flow from the opposing compartments

to be focused to the central core of the compartment at the downstream portion, but not in the upstream portion suggesting a flow of PBS from side compartments (Fig. 2d).

Table 1 Tracer diffusion during axotomy

\begin{tabular}{llll}
\hline & $2 \mathrm{~min}$ & $5 \mathrm{~min}$ & $10 \mathrm{~min}$ \\
\hline Somatic compartment & $0.02 \pm 0.29$ & $0.18 \pm 0.30$ & $0.29 \pm 0.31$ \\
Central compartment & $68.4 \pm 9.8$ & $58.1 \pm 3.9$ & $44.9 \pm 5.8$ \\
\hline
\end{tabular}

Tracer concentration is linearly related to the fluorescent signal $\left(R^{2}>0.99\right)$. Values are given in percentage of maximum concentration and represent the averages of three circuits where three regions were imaged per compartment. $100 \%$ tracer is added to the central compartment at zero time point. It is evident that during axotomy simulation, the tracer does not diffuse to the somatic or axonal compartments; instead, it is diluted by the influx from these compartments 
Table 2 Tracer diffusion during long-term treatment

\begin{tabular}{lllllr}
\hline & $0 \mathrm{~h}$ & $1 \mathrm{~h}$ & $2 \mathrm{~h}$ & $4 \mathrm{~h}$ & $8 \mathrm{~h}$ \\
\hline Axonal compartment & $83.2 \pm 7.91$ & $72.8 \pm 0.52$ & $66.7 \pm 0.55$ & $76.1 \pm 4.79$ & $69.4 \pm 10.7$ \\
Central compartment & $1.41 \pm 1.36$ & $4.23 \pm 4.25$ & $13.3 \pm 0.70$ & $13.5 \pm 0.11$ & $13.1 \pm 0.60$ \\
Somatic compartment & $0.26 \pm 0.13$ & $0.34 \pm 0.13$ & $0.32 \pm 0.02$ & $0.37 \pm 0.15$ & $0.26 \pm 0.00$ \\
\hline
\end{tabular}

Tracer concentration is linearly related to the fluorescent signal $\left(R^{2}>0.99\right)$. Values are given in percentage of maximum concentration and represent the averages of three circuits where three regions were imaged per compartment. $100 \%$ tracer is added to the axonal compartment at zero time point. It is evident that the tracer reaches a steady concentration at the central compartment but cannot reach the somatic compartment

The axotomy procedure was also simulated by using two different tracer molecules in order to visualize the flow dynamics of both the detergent solution and the medium in the side compartments. The volume configuration described above was used where the side compartments contained blue tracer and the detergent solution contained red tracer. It is evident that the detergent solution flushes out the medium in the central compartment and completely fills it in less than 10s following its placement to the top reservoir (Supplementary Movie 2). In the downstream portion of the central compartment where the pressure gradient is highest, the blue tracer from side compartments can be seen to flow into the central compartment over time (Supplementary Movie 3).

Additional experiments were conducted to measure the extent of particle diffusion from one side compartment to the other side compartment during long term treatments. Equal liquid volumes were applied to each reservoir, and the tracer was added to the distal compartment. Starting with $2 \mathrm{~h}$ time point, a substantial diffusion of the tracer to the central compartment (up to $18 \%$ of the maximal concentration) was observed; however, no tracer was detected in the somatic compartment (Table 2), suggesting that the central compartment acted as a sink for long-term particle diffusion between the two side compartments.

Collectively, these experiments demonstrate that in the timeframe of axotomy experiments, the flow of the detergent-containing medium through the central compartment during axotomy is confined to this compartment due to pressure-driven inflow through the microchannels. Also, the long-term diffusion of molecules from axonal compartment is maintained within the central compartment and cannot reach the opposite somatic compartment, thus enabling us to treat axonal and somatic compartments independently.

\section{Cortical Neuron Culture in Three-compartmental Microfluidic Devices}

Neurons isolated from embryonic mouse cortex were seeded at a relatively high cell density. Approximately $5 \%$ of the cells seeded in the somatic compartment after 8 days of culture were found to be astrocytes, assessed by GFAP staining (not shown). Each circuit involved 167 parallel microchannels, and allowed the crossing of hundreds of axons to the central compartment. Most of these axons continued their extension and reached the distal compartment, forming a total length of 1.5-2 mm (Fig. 3). $38.3 \pm$ $3.4 \%$ of the axons ( $N=11$ control circuits) that reached the central compartment also reached the axonal compartment by 8 days in vitro. The length of the central compartment is critical for efficient invasion of the distal compartment by axons. Axons tend to fasciculate within microchannels, but when entering the central compartment, they slightly splay apart and divert from their trajectories (Fig. 3a). A compartment length of $100 \mu \mathrm{m}$ was found suitable for allowing the entrance of a large portion of the axons into the second set of microchannels.

\section{Central Axotomy Induces Growth Cone Collapse and Wallerian-like Distal Degeneration}

Central axotomy was conducted by a brief pulse of $0.1 \%$ saponin flowing over the central compartment. This corresponds to a segment along the axons at most $100 \mu \mathrm{m}$ long depending on the position in the central compartment (due to the progressive confinement of the saponin flow by liquid exiting from the microchannels), and at least $450 \mu \mathrm{m}$ away from their cell bodies. Axotomy causes complete removal of the central segment of axons (Fig. 3b). Axotomy induced progressive distal degeneration in the axon, leading to axonal bead formation and fragmentation, whereas cell bodies did not show any morphological alterations (Fig. 4a). Distal axonal fragmentation was quantified at different post-injury time points by analyzing tubulin staining images of fixed axons. In the axotomy group, the fragmentation index increased significantly between all time points studied, before reaching a plateau at $8 \mathrm{~h}$. Starting with $4 \mathrm{~h}$ time point, axonal fragmentation was significantly higher in the axotomy group compared to sham controls, which underwent the exact protocol except that there was no saponin in the medium flown through the central compartment (Fig. 4b).

Axonal growth cones are indicative of elongating axons, and normally exhibit a characteristic morphology where actin filaments form thin filopodia and veil-like lamellipodia 


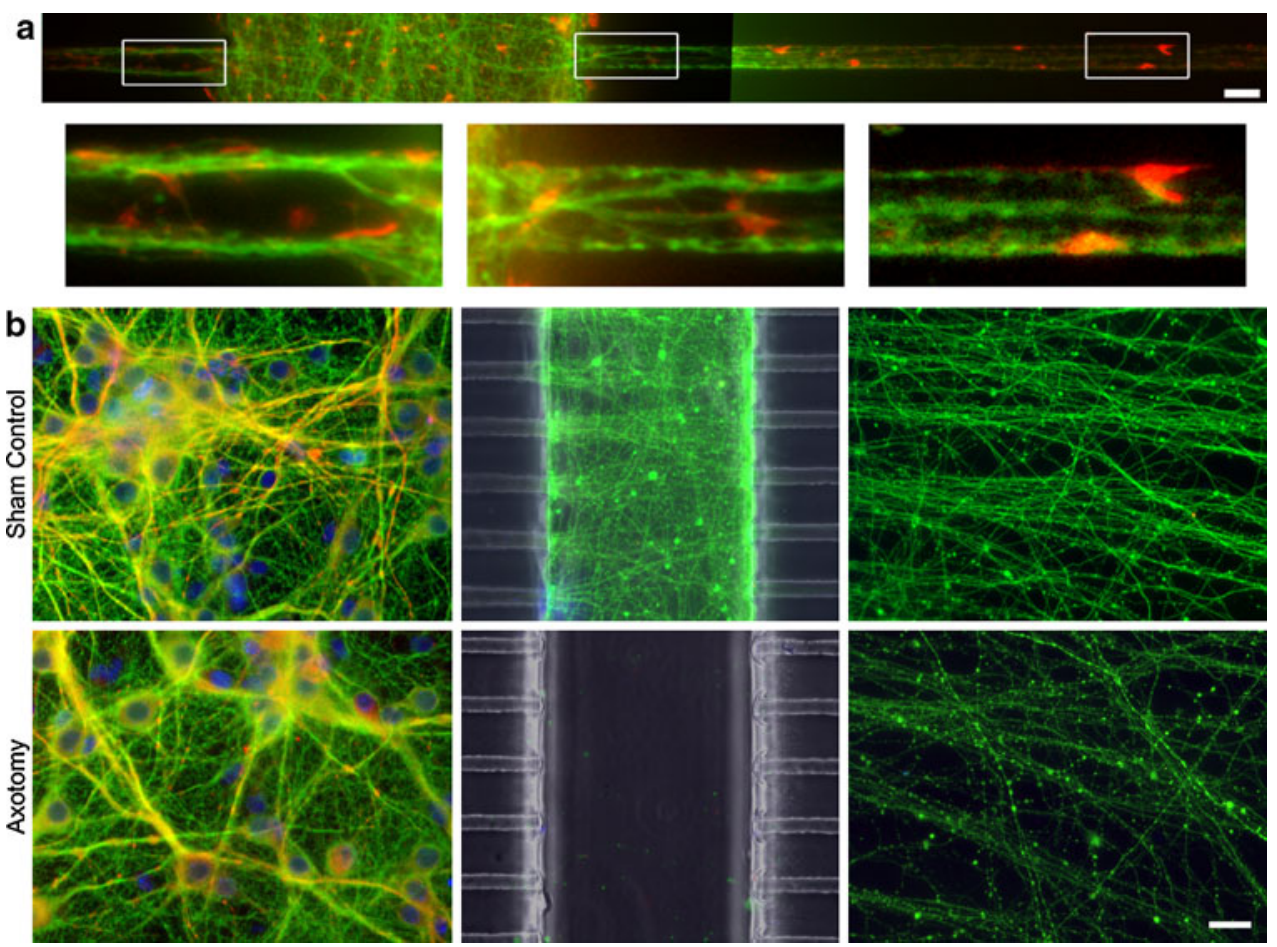

Fig. 3 (a) Cortical neuron morphology within microchannels and central compartment. Neurons were stained against $\beta$-3-tubulin (green) and F-actin (red). The variation in signal intensity is due to the variation in staining efficiency within microchannels. Axons usually follow the edges of microchannels and form bundles that splay apart at the entry to the compartment. Scale bar $=10 \mu \mathrm{m}$. Boxed regions are $3.6 \times$ magnified and their contrast is enhanced. (b)
Sham control (top) and axotomized (bottom) neurons in threecompartmental microfluidic circuits. Somatic and axonal images are overlays of $\beta$-3-tubulin (green), MAP2 (red), and Hoechst (blue) staining; central images have also phase contrast image in the overlay. Axotomy results in complete destruction of the central segment of the axons; however, neuronal integrity is well preserved in both somatic and axonal compartments. Scale bar $=20 \mu \mathrm{m}$
(Gallo and Letourneau 2004). In control neurons, growth cones are abundant and display characteristic actin-dense morphology; in axotomized neurons, in contrast, actin staining is less concentrated at the tips, indicating the collapse of the growth cone (Fig. 5a). $1 \mathrm{~h}$ after axotomy, the axons are structurally intact but the number of growth cones normalized by the total axon mass is found to be significantly lower compared to the control cultures (Fig. 5a).

In vivo, axotomy-induced distal axon degeneration has been shown to be heterogeneous in timing: At a given time point, simultaneously severed axons showed distinct morphologies, ranging from intact axons to complete fragmentation (Beirowski et al. 2005). In our experiments, at early time points following axotomy, we observed intact, beaded, and completely fragmented axons in the same distal compartment, suggesting asynchronous degeneration of distal axons (Fig. 5b). In vivo, axotomy-induced Wallerian degeneration has been demonstrated to progress in the anterograde direction and in a wave-like fashion within individual axons (Beirowski et al. 2005). At $8 \mathrm{~h}$ following axotomy, we observed a fraction of degenerating axons that are completely fragmented in the proximal segment, beaded in the central segment and intact in the distal segment (Fig. 5c). These distinct regions with characteristic morphologies displayed in the same distal axon stump may be indicative of anterograde progression of the degeneration "wave" in our axotomy model.

Distal Degeneration is Delayed by $\beta$ NAD Protection but Not by Caspase or Calpain Inhibition

Early calpain activation in Wallerian degeneration was shown to precede morphological events in both in vivo and in vitro model systems (Glass et al. 2002). Caspases, on the other hand, have been shown to be activated in cell bodies of axotomized neurons but not in distal axon segments undergoing Wallerian degeneration (Finn et al. 2000). We tested whether axotomy-induced distal axon fragmentation could be blocked by inhibiting local calpain or caspase activity. Post-injury axonal calpain and caspase activity was inhibited using specific inhibitors of these proteases, ALLN and z-VAD, respectively. We first determined that axotomy induces calpain activity and that this activity can be blocked by ALLN, by using a reporter molecule that fluoresces when specifically cleaved by calpain 


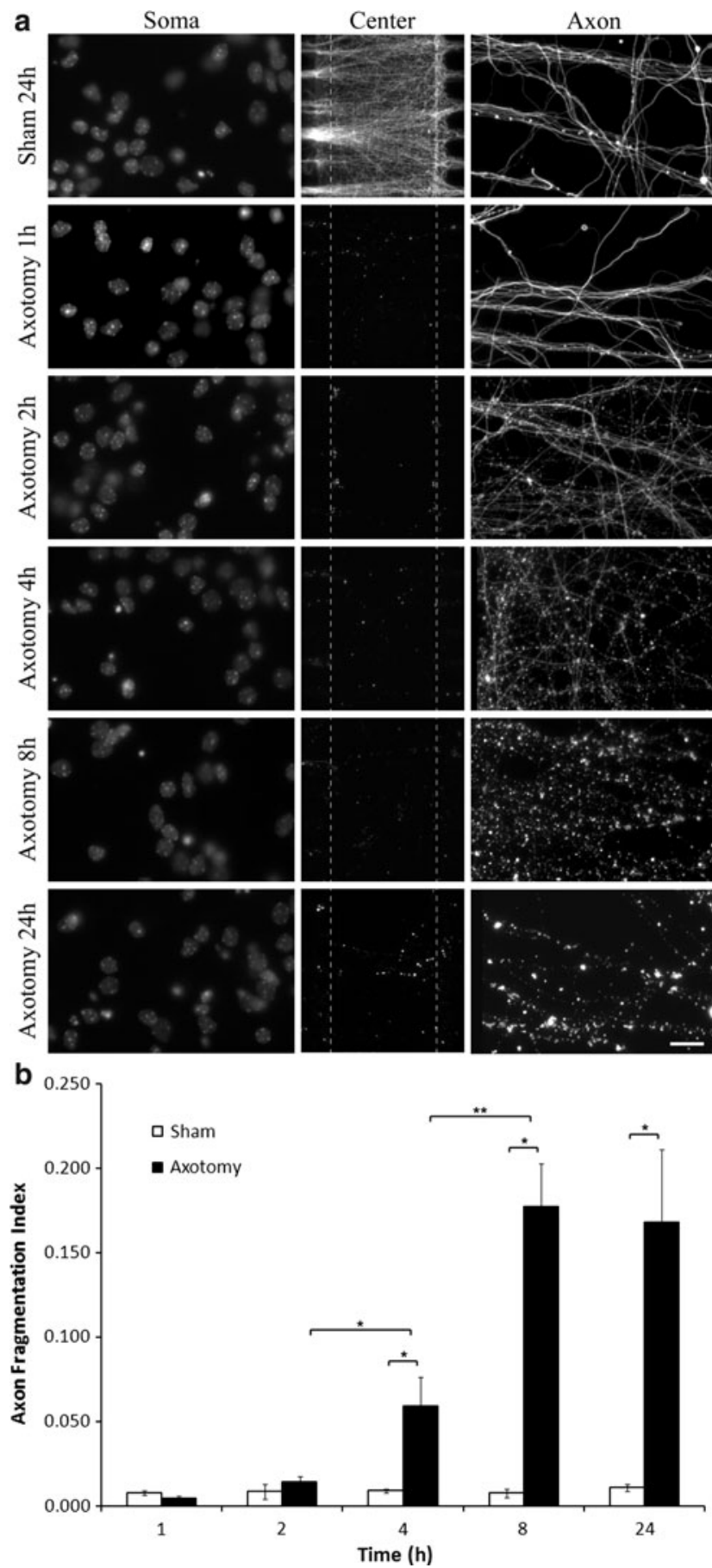

Fig. 4 Axonal degeneration and somatic survival following central axotomy to cortical neurons. (b) Example areas showing somatic (Hoechst staining) central, and axonal compartments ( $\beta$-3-tubulin staining) at different time points post-injury. Axotomy induced progressive distal degeneration leading to axonal beading and microtubule fragmentation. Scale bar $=20 \mu \mathrm{m}$. (b) Axonal fragmentation index is significantly higher than controls starting with $4 \mathrm{~h}$ time point. An index value above 0.15 corresponds to the complete fragmentation of distal axons. Dark bars represent axotomized cultures; light bars represent sham controls. $N=3$ independent circuits for axotomy; $N=2$ independent circuits for sham controls. Error bars denote SEM. $* p<0.05$; $* * p<0.01$; ANOVA is followed by $t$-test
(Supplementary Figure 2). We then conducted timed experiments to test the effect of local treatments. Distal axonal fragmentation was not affected by either calpain or caspase inhibition (Fig. 6).

In vitro Wallerian degeneration of axons of DRG explants were significantly delayed when these neurons were pretreated with $\beta \mathrm{NAD}$ for at least $24 \mathrm{~h}$ (Araki et al. $2004)$. To test whether and to what extent $\beta$ NAD pretreatment protects axotomy-induced degeneration in our model system, we treated neurons with $5 \mathrm{mM} \beta \mathrm{NAD}$ starting 2 days prior to axotomy and continuing thereafter. Distal axonal fragmentation was significantly blocked in $\beta$ NAD treated neurons until the $8 \mathrm{~h}$ time point; however, no significant protection is evident at the $24 \mathrm{~h}$ time point (Fig. 6). Post-axotomy treatment of neurons with $\beta \mathrm{NAD}$ did not provide any protection against axonal fragmentation (not shown). Viability of axotomized neurons with or without treatment was not significantly different than that of the sham controls at $24 \mathrm{~h}$ time point, indicating that distal degeneration is the major consequence of central axotomy (Supplementary Figure 3).

\section{Discussion}

Simultaneous axotomy of a large number of primary neurons may provide an interesting in vitro model system to study molecular mechanisms of axonal degeneration in a strictly controlled environment and a highly scalable fashion. Fluidic separation of proximal and distal neuronal compartments prior to and following axotomy enables exclusive access to somata and axons, allowing a variety of injury paradigms and therapeutic approaches to be tested. Such a model system could also be useful in the screening of pharmacological agents that target neurodegenerative mechanisms, using well-characterized paradigms such as Wallerian degeneration. Moreover, this model system can also be used for the local application of many soluble substances, with multiple potential uses in neuroscience field. In this study, we induced localized axotomy to primary central neurons and characterized distal axonal degeneration. Axotomy induced Wallerian-like, asynchronous, progressive distal degeneration that could be delayed by $\beta$ NAD pretreatment; whereas post-axotomy inhibition of distal calpain or caspase activity did not affect the final outcome.

Subcellular compartmentalization of neurons using microfluidic systems was previously described (Taylor et al. 2005). The fluidic isolation of a single compartment was demonstrated over a 20 -h period by using fluorescent dextran and radioactive methionine as markers; however, the extent of dilution of the tracer molecules by the flow from the over-pressurized compartment was not reported in that study (Taylor et al. 2005). We performed a flow mapping of 


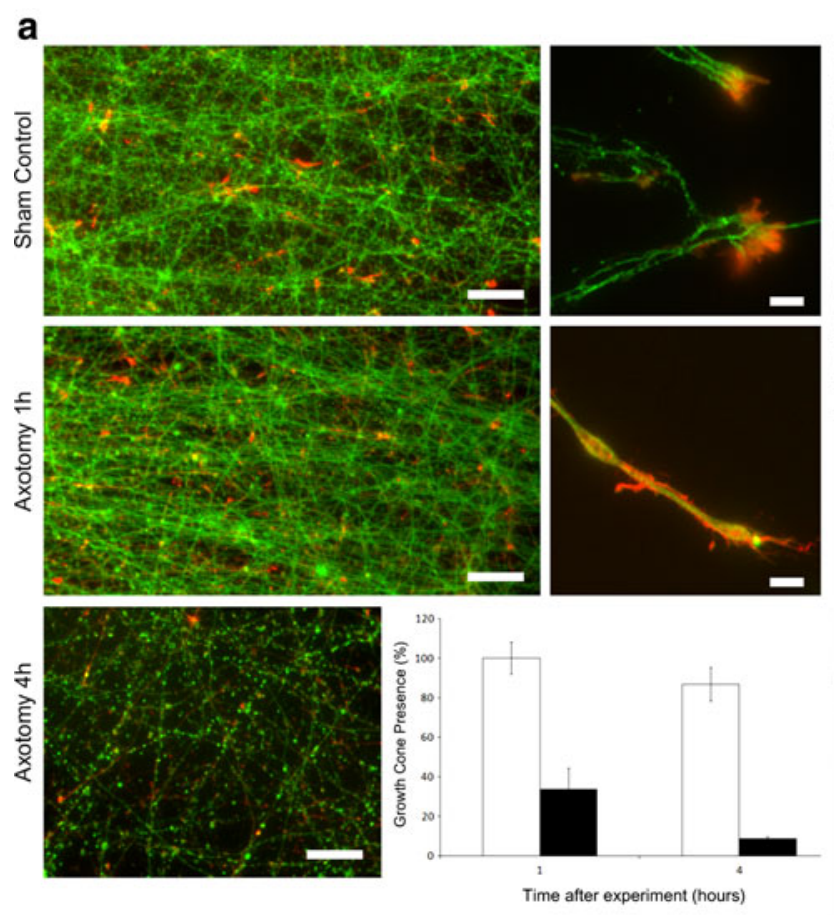

Fig. 5 (a) Actin and tubulin co-staining shows early growth cone collapse following axotomy. The number of established growth cones decreases significantly $1 \mathrm{~h}$ following axotomy, even though the axonal cytoskeleton is intact. Actin filaments are concentrated at the tips of intact axons whereas a more diffuse distribution is observed at the tips of cut axons. Scale bars $=20 \mu \mathrm{m}$ (left panels) and $5 \mu \mathrm{m}$ (right panels). (b) Heterogeneity of axotomy-induced distal degeneration can be seen at $4 \mathrm{~h}$ post-axotomy. Coexistence of intact (Luo and O'Leary 2005), beaded (Houle and Tessler 2003), and completely fragmented (Griffin et al. 1995) axons suggests asynchronous distal degeneration of axons which underwent simultaneous axotomy at

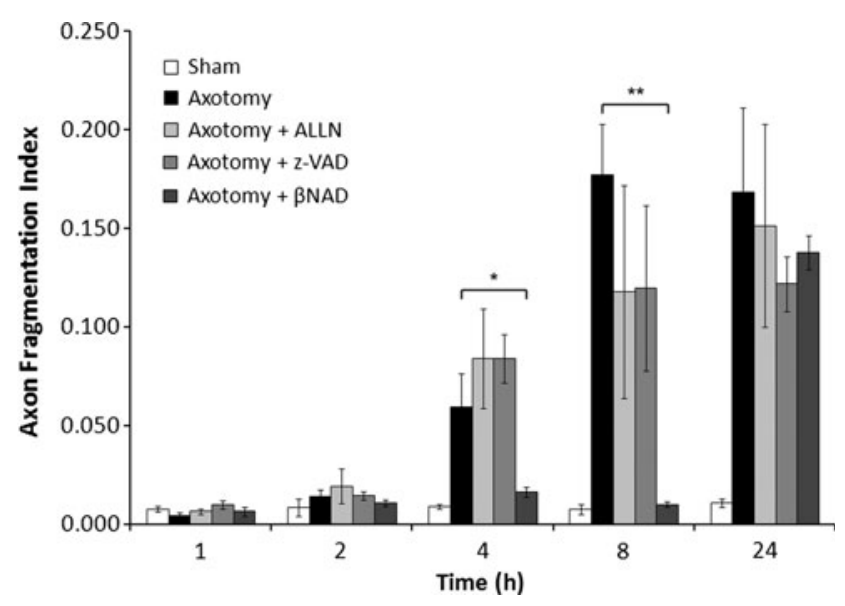

Fig. 6 Axonal fragmentation indices were calculated for different experimental groups at each time point. An index value above 0.15 corresponds to the complete fragmentation of distal axons. Postaxotomy treatment of distal axons with calpain inhibitor ALLN $(10 \mu \mathrm{M})$ or caspase inhibitor z-VAD $(20 \mu \mathrm{M})$ did not provide any protection. Pretreatment with $\beta$ NAD $(5 \mathrm{mM})$ delayed axotomyinduced increase in fragmentation. Error bars represent SEM. ANOVA is followed by $t$-test. $* p<0.05$, ** $p<0.01$ b
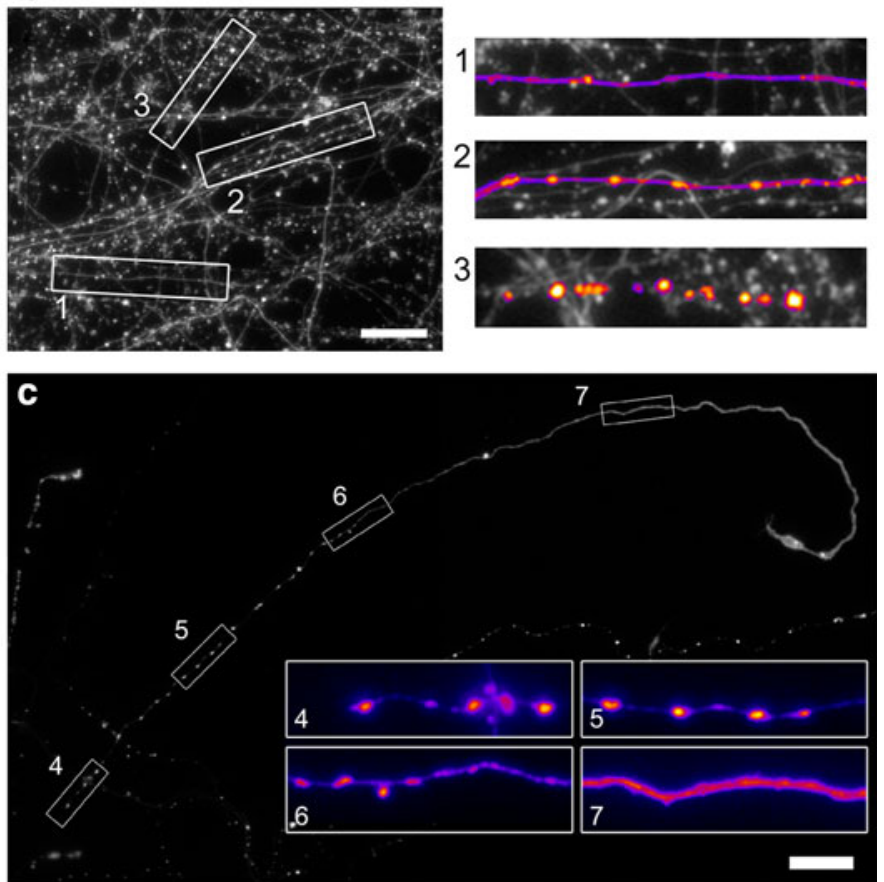

approximately same distance from their cell bodies. Boxed areas are $2 \times$ magnified and pseudo-colored images of the axons of interest are superposed. Scale bar $=50 \mu \mathrm{m}$. (c) Anterograde progression of axonal degeneration at $8 \mathrm{~h}$ following central axotomy. The axon distal to the axotomy site is completely fragmented except the most distal segment shown. The proximal portion of this segment exhibits heavy beading (insets 4 and 5), whereas the distal portion is intact (inset 7). A short transition zone (inset 6) separates beaded and intact regions of the axon, suggesting a wave-like anterograde progression of degeneration. Boxed areas are $4 \times$ magnified and pseudo-colored to display axonal continuity. Scale bar $=100 \mu \mathrm{m}$

the detergent solution through the central compartment and examined if the detergent could diffuse to the side compartments. Our observation that the flow is restricted to the central core of the central compartment, especially in the downstream portion of the compartment, suggests that a dilution of the detergent due to the flow from the overpressurized side compartments. However, we have not observed any local variation in the distal compartment along the detergent flow direction. Cumulatively, simulation results suggest that central axotomy is well confined in the central compartment. On the other hand, long-term fluidic isolation of the furthermost compartment (i.e., somatic compartment in the case of axonal treatment) is shown to be maintained for at least $8 \mathrm{~h}$. The dye used in these simulations interacted with PDMS polymer and limited diffusion studies to relatively short periods of time $(\sim 8 \mathrm{~h})$. However, as the diffusion of the dye to the central compartment was stabilized at $2 \mathrm{~h}$ time point, we are confident that the central compartment acted as a sink and diffusing dye molecules never reached the somatic compartment. Diffusion and dilution of molecules are 
potential problems for long-term isolated treatment experiments in microfluidic soma-axon compartmentalization systems; because the inter-compartmental flow necessary to avoid diffusion dilutes the treatment especially near microchannel exits (Sutton et al. 2007). Thus the three-compartmental design proposed here could also be used to stabilize these systems in terms of fluidic isolation, and ensuring that neither the proximal nor the distal compartment be contaminated by solution from the other one. Alternatively, a pressure control device or a syringe pump may be used to impose continuous pressure gradients between compartments such that the steady flow prevents molecules to diffuse upstream and the dilution is avoided by constantly circulating the medium containing the treatment.

Wallerian degeneration, degeneration of the distal stump of a transected axon, is a well-described phenomenon (Waller 1850; Coleman 2005) that receives growing interest since the recent discovery of a mutant mouse strain which exhibits slow Wallerian degeneration (Glass et al. 1993; Coleman 2005). A longitudinal in vivo study examining fragmentation patterns of individual axons following transection and nerve crush revealed the progressive and directional nature of Wallerian degeneration. Peripheral neurons that were transected simultaneously exhibited both intact and completely fragmented distal axons, indicating that Wallerian degeneration is an asynchronous process (Beirowski et al. 2005; Kerschensteiner et al. 2005). Transected axons exhibited a long latency period followed by a rapid degeneration phase, suggesting a wave of fragmentation travelling in the anterograde direction from the axotomy site (Beirowski et al. 2005; Kerschensteiner et al. 2005). Our results show that at a given time following axotomy distal axons display very different morphologies varying from intact to completely fragmented microtubule cytoskeleton. We also observed distal axon stumps which are completely fragmented in the proximal segment, beaded in the middle segment and intact at the distal segment, suggesting an anterograde progression of axon degeneration. Cumulatively, these observations indicate that CNS neurons undergoing local axotomy in our novel microfluidic culture system exhibit characteristic morphological changes similar to those observed during in vivo Wallerian degeneration. However, axotomy-induced degeneration occurs faster when compared to in vivo Wallerian degeneration in the CNS (Aguayo et al. 1991; Kerschensteiner et al. 2005) and in vitro Wallerian degeneration in the PNS (MacInnis and Campenot 2005). Several reasons could account for this enhanced vulnerability of cultured axons: (i) they are reminiscent of developing immature axons as they grow indefinitely in the absence of a target; (ii) they might lack necessary trophic factors due to the absence of post-synaptic neurons and supporting neuroglia; (iii) this shorter degeneration could be a more straightforward consequence of the shorter length of our cultured axons, as compared to in vivo ones.

Calpains are $\mathrm{Ca}^{2+}$-dependent cysteine proteases having multiple physiological functions (Liu et al. 2008). They are known to degrade cytoskeletal proteins such as microtubule-associated proteins, spectrin, and tubulin (Billger et al. 1988; Johnson et al. 1991). Early activation of calpain is believed to contribute in the progressive structural damage in the axonal cytoskeleton following traumatic brain injury, whereas the delayed activation is attributed to the late phases of axonal degeneration (Saatman et al. 2003). Axotomy-induced increase in the intracellular $\mathrm{Ca}^{2+}$ concentration causes early calpain activation and subsequent degeneration of the cytoskeleton (Glass et al. 2002). Inhibition of calpain activity has been shown to inhibit distal axon degeneration following axotomy in DRG explants (Wang et al. 2000). In vivo results revealed that calpain inhibition blocks early (30 $\mathrm{min}$ ) changes induced by axotomy (Kerschensteiner et al. 2005); however, in vitro results suggested that calpain inhibition can only delay axonal degeneration for approximately $4 \mathrm{~h}$ (Zhai et al. 2003). In our model, inhibition of distal calpain activity following axotomy did not protect axons, even though it blocked early calpain activity induced by axotomy. Axotomy may be inducing multiple degenerative pathways that have different timeframes. In in vitro models, such as ours, the pace of axonal degeneration may be too fast to be protected by calpain inhibition. Caspases, a family of cysteine proteases essential in apoptosis, were shown to not be involved in Wallerian degeneration (Finn et al. 2000). Our finding that caspase inhibition does not alter axotomy-induced distal fragmentation is consistent with the literature. We observed rapid growth cone collapse following axotomy, and significant reduction in active growth cones was observed well before cytoskeletal fragmentation was evident. This observation suggests a dual degeneration mechanism following axotomy, in which a rapid signal travels towards the tips of axons and induces growth cone collapse, whereas a secondary, delayed mechanism causes cytoskeletal breakdown. However, the molecular mechanisms responsible for these fast and delayed effects remain to be elucidated.

Studies using the slow Wallerian degeneration mutant mouse $\left(\mathrm{Wld}^{\mathrm{S}}\right)$, where the Wallerian degeneration is significantly delayed due to the expression of a nuclear fusion protein (Wld ${ }^{\mathrm{S}}$ protein), suggested that Wallerian degeneration is an active process of axonal self-destruction (Raff et al. 2002). The chimeric Wld ${ }^{\mathrm{S}}$ protein functions like the nuclear $\beta$ NAD-producing enzyme nicotinamide mononucleotide adenylyl transferase 1 (Nmnat1); hence, WldS mice have enhanced $\beta$ NAD biosynthesis. Over-expression of Nmnat 1 has been shown to protect axons following in vitro (Araki et al. 2004) but not in vivo axotomy (Conforti et al. 2007; Yahata et al. 2009). Recent studies revealed that 
Nmnat3, the mitochondrial isoform of Nmnat1, protects transected neurons as much as Wld ${ }^{\mathrm{S}}$ (Avery et al. 2009) and that extra-nuclear $\mathrm{Wld}^{\mathrm{S}}$ protects neurons more than the native Wld ${ }^{\text {S }}$ (Beirowski et al. 2009). These findings highlight the importance of subcellular compartmentalization in neurodegeneration. Our observation that $\beta$ NAD pretreatment successfully delays axotomy-induced distal degeneration is in cohort with previous reports (Araki et al. 2004), and suggests that increased bioenergetic levels prior to axotomy could be the critical factor in the protection. Our in vitro axotomy model enables temporal and spatial examination of molecular mechanisms of neurodegeneration; therefore, it can be a promising tool for investigating a variety of neuroprotective mechanisms, including $\beta \mathrm{NAD}$ pretreatment, in the future.

\section{Summary}

We applied simultaneous axotomy to a large number of parallel axons of cultured primary central neurons. Results show that our novel in vitro axotomy model repeats characteristic features of in vivo Wallerian degeneration such as directional, progressive, asynchronous distal axon fragmentation, protection with $\beta \mathrm{NAD}$ pretreatment, and independence of caspase pathway. Our novel model system relies on microfluidic isolation and enables exclusive access to proximal and distal compartments. It is thus a powerful tool to study spatial and temporal aspects of molecular mechanisms in neurodegeneration.

Acknowledgments This study was supported by a grant from the French National Research Agency (Project Neurofluidique, ANR-06Neuro-032-01). The authors thank Ms. Laure Hirsch and Mr. Hugo Caicedo for technical help.

Open Access This article is distributed under the terms of the Creative Commons Attribution Noncommercial License which permits any noncommercial use, distribution, and reproduction in any medium, provided the original author(s) and source are credited.

\section{References}

Aguayo AJ, Rasminsky M, Bray GM, Carbonetto S, McKerracher L, Villegas-Perez MP et al (1991) Degenerative and regenerative responses of injured neurons in the central nervous system of adult mammals. Philos Trans R Soc Lond B Biol Sci 331(1261): 337-343

Ambron RT, Dulin MF, Zhang XP, Schmied R, Walters ET (1995) Axoplasm enriched in a protein mobilized by nerve injury induces memory-like alterations in Aplysia neurons. J Neurosci 15(5 Pt 1):3440-3446

Araki T, Sasaki Y, Milbrandt J (2004) Increased nuclear NAD biosynthesis and SIRT1 activation prevent axonal degeneration. Science 305(5686):1010-1013
Avery MA, Sheehan AE, Kerr KS, Wang J, Freeman MR (2009) Wld $S$ requires Nmnat 1 enzymatic activity and N16-VCP interactions to suppress Wallerian degeneration. J Cell Biol 184(4):501513

Beirowski B, Adalbert R, Wagner D, Grumme DS, Addicks K, Ribchester RR et al (2005) The progressive nature of Wallerian degeneration in wild-type and slow Wallerian degeneration (WldS) nerves. BMC Neurosci 6:6

Beirowski B, Babetto E, Gilley J, Mazzola F, Conforti L, Janeckova L et al (2009) Non-nuclear Wld(S) determines its neuroprotective efficacy for axons and synapses in vivo. J Neurosci 29(3):653668

Billger M, Wallin M, Karlsson JO (1988) Proteolysis of tubulin and microtubule-associated proteins 1 and 2 by calpain I and II. Difference in sensitivity of assembled and disassembled microtubules. Cell Calcium 9(1):33-44

Bossy-Wetzel E, Schwarzenbacher R, Lipton SA (2004) Molecular pathways to neurodegeneration. Nat Med 10(Suppl):S2-S9

Buki A, Povlishock JT (2006) All roads lead to disconnection?Traumatic axonal injury revisited. Acta Neurochir (Wien) 148(2): 181-193 discussion 93-4

Chung BG, Flanagan LA, Rhee SW, Schwartz PH, Lee AP, Monuki ES et al (2005) Human neural stem cell growth and differentiation in a gradient-generating microfluidic device. Lab Chip 5(4):401-406

Coleman M (2005) Axon degeneration mechanisms: commonality amid diversity. Nat Rev Neurosci 6(11):889-898

Conforti L, Fang G, Beirowski B, Wang MS, Sorci L, Asress S et al (2007) $\mathrm{NAD}(+)$ and axon degeneration revisited: Nmnat1 cannot substitute for Wld(S) to delay Wallerian degeneration. Cell Death Differ 14(1):116-127

El-Ali J, Sorger PK, Jensen KF (2006) Cells on chips. Nature 442(7101): 403-411

Fan YW, Cui FZ, Hou SP, Xu QY, Chen LN, Lee IS (2002) Culture of neural cells on silicon wafers with nano-scale surface topograph. J Neurosci Methods 120(1):17-23

Finn JT, Weil M, Archer F, Siman R, Srinivasan A, Raff MC (2000) Evidence that Wallerian degeneration and localized axon degeneration induced by local neurotrophin deprivation do not involve caspases. J Neurosci 20(4):1333-1341

Francisco H, Yellen BB, Halverson DS, Friedman G, Gallo G (2007) Regulation of axon guidance and extension by three-dimensional constraints. Biomaterials 28(23):3398-3407

Gallo G (2004) Myosin II activity is required for severing-induced axon retraction in vitro. Exp Neurol 189(1):112-121

Gallo G, Letourneau PC (1999) Different contributions of microtubule dynamics and transport to the growth of axons and collateral sprouts. J Neurosci 19(10):3860-3873

Gallo G, Letourneau PC (2004) Regulation of growth cone actin filaments by guidance cues. J Neurobiol 58(1):92-102

Glass JD, Brushart TM, George EB, Griffin JW (1993) Prolonged survival of transected nerve fibres in C57BL/Ola mice is an intrinsic characteristic of the axon. J Neurocytol 22(5):311-321

Glass JD, Culver DG, Levey AI, Nash NR (2002) Very early activation of $\mathrm{m}$-calpain in peripheral nerve during Wallerian degeneration. J Neurol Sci 196(1-2):9-20

Griffin JW, George EB, Hsieh S, Glass JD (1995) Axonal degeneration and disorders of the axonal cytoskeleton. In: Waxman SG, Kocsis JD, Stys PK (eds) The axon: structure, function and pathophysiology. Oxford University Press, New York, pp 375-390

Hanz S, Perlson E, Willis D, Zheng JQ, Massarwa R, Huerta JJ et al (2003) Axoplasmic importins enable retrograde injury signaling in lesioned nerve. Neuron 40(6):1095-1104

Houle JD, Tessler A (2003) Repair of chronic spinal cord injury. Exp Neurol 182(2):247-260 
Johnson GV, Litersky JM, Jope RS (1991) Degradation of microtubule-associated protein 2 and brain spectrin by calpain: a comparative study. J Neurochem 56(5):1630-1638

Kerschensteiner M, Schwab ME, Lichtman JW, Misgeld T (2005) In vivo imaging of axonal degeneration and regeneration in the injured spinal cord. Nat Med 11(5):572-577

Kilinc D, Gallo G, Barbee KA (2009) Mechanical membrane injury induces axonal beading through localized activation of calpain. Exp Neurol 219(2):553-561

Kim Y, Karthikeyan K, Chivri S, Dave DP (2009) Neuro-optical microfluidic platform to study injury and regeneration of single axons. Lab Chip 9(17):2576-2581

Kuhn MJ, Mikulis DJ, Ayoub DM, Kosofsky BE, Davis KR, Taveras JM (1989) Wallerian degeneration after cerebral infarction: evaluation with sequential MR imaging. Radiology 172(1):179182

Liu J, Liu MC, Wang KK (2008) Calpain in the CNS: from synaptic function to neurotoxicity. Sci Signal 1(14):re1

Luo L, O'Leary DD (2005) Axon retraction and degeneration in development and disease. Annu Rev Neurosci 28:127-156

MacInnis BL, Campenot RB (2005) Regulation of Wallerian degeneration and nerve growth factor withdrawal-induced pruning of axons of sympathetic neurons by the proteasome and the MEK/ Erk pathway. Mol Cell Neurosci 28(3):430-439

Mandolesi G, Madeddu F, Bozzi Y, Maffei L, Ratto GM (2004) Acute physiological response of mammalian central neurons to axotomy: ionic regulation and electrical activity. FASEB J 18(15): 1934-1936

Morales R, Riss M, Wang L, Gavin R, Del Rio JA, Alcubilla R et al (2008) Integrating multi-unit electrophysiology and plastic culture dishes for network neuroscience. Lab Chip 8(11):18961905

Morin F, Nishimura N, Griscom L, Lepioufle B, Fujita H, Takamura $\mathrm{Y}$ et al (2006) Constraining the connectivity of neuronal networks cultured on microelectrode arrays with microfluidic techniques: a step towards neuron-based functional chips. Biosens Bioelectron 21(7):1093-1100

Nakashima Y, Yasuda T (2007) Cell differentiation guidance using chemical stimulation controlled by a microfluidic device. Sens Actuator A: Phys 139(1-2):252-258

Otsu N (1979) A threshold selection method from gray level histograms. IEEE Trans Syst Man Cybern 9:62-66

Pearce TM, Wilson JA, Oakes SG, Chiu SY, Williams JC (2005) Integrated microelectrode array and microfluidics for temperature clamp of sensory neurons in culture. Lab Chip 5(1):97-101

Raff MC, Whitmore AV, Finn JT (2002) Axonal self-destruction and neurodegeneration. Science 296(5569):868-871

Ravula SK, Wang MS, McClain MA, Asress SA, Frazier B, Glass JD (2007) Spatiotemporal localization of injury potentials in DRG neurons during vincristine-induced axonal degeneration. Neurosci Lett 415(1):34-39

Saatman KE, Abai B, Grosvenor A, Vorwerk CK, Smith DH, Meaney DF (2003) Traumatic axonal injury results in biphasic calpain activation and retrograde transport impairment in mice. J Cereb Blood Flow Metab 23(1):34-42

Sato K, Egami A, Odake T, Tokeshi M, Aihara M, Kitamori T (2006) Monitoring of intercellular messengers released from neuron networks cultured in a microchip. J Chromatogr A 1111(2): $228-232$

Singleton RH, Zhu J, Stone JR, Povlishock JT (2002) Traumatically induced axotomy adjacent to the soma does not result in acute neuronal death. J Neurosci 22(3):791-802

Sutton MA, Taylor AM, Ito HT, Pham A, Schuman EM (2007) Postsynaptic decoding of neural activity: eEF2 as a biochemical sensor coupling miniature synaptic transmission to local protein synthesis. Neuron 55(4):648-661

Taylor AM, Blurton-Jones M, Rhee SW, Cribbs DH, Cotman CW, Jeon NL (2005) A microfluidic culture platform for CNS axonal injury, regeneration and transport. Nat Methods 2(8):599-605

Vahidi B, Park JW, Kim HJ, Jeon NL (2008) Microfluidic-based strip assay for testing the effects of various surface-bound inhibitors in spinal cord injury. J Neurosci Methods 170(2):188-196

Waller A (1850) Experiments on the section of the glossopharyngeal and hypoglossal nerves of the frog, and observations of the alterations produced thereby in the structure of their primitive fibres. Philos Trans R Soc Lond B 140:423-429

Wang MS, Wu Y, Culver DG, Glass JD (2000) Pathogenesis of axonal degeneration: parallels between Wallerian degeneration and vincristine neuropathy. J Neuropathol Exp Neurol 59(7): 599-606

Weibel DB, Garstecki P, Whitesides GM (2005) Combining microscience and neurobiology. Curr Opin Neurobiol 15(5):560-567

Weibel DB, Diluzio WR, Whitesides GM (2007) Microfabrication meets microbiology. Nat Rev Microbiol 5(3):209-218

Willaime S, Vanhoutte P, Caboche J, Lemaigre-Dubreuil Y, Mariani J, Brugg B (2001) Ceramide-induced apoptosis in cortical neurons is mediated by an increase in p38 phosphorylation and not by the decrease in ERK phosphorylation. Eur $\mathrm{J}$ Neurosci 13(11):2037-2046

Yahata N, Yuasa S, Araki T (2009) Nicotinamide mononucleotide adenylyltransferase expression in mitochondrial matrix delays Wallerian degeneration. J Neurosci 29(19):6276-6284

Yanik MF, Cinar H, Cinar HN, Chisholm AD, Jin Y, Ben-Yakar A (2004) Neurosurgery: functional regeneration after laser axotomy. Nature 432(7019):822

Zhai Q, Wang J, Kim A, Liu Q, Watts R, Hoopfer E et al (2003) Involvement of the ubiquitin-proteasome system in the early stages of wallerian degeneration. Neuron 39(2):217-225 